[0212-7199 (2006) 23: 7; pp 329-330] ANALES DE MEDICINA INTERNA Copyright (C) 2006 ARAN EDICIONES, S.L.

AN. MED. InTERNA (Madrid) Vol. 23, N. $^{\circ}$ 7, pp. $329-330,2006$

\title{
Uracosinus: una causa poco frecuente de onfalitis recurrente en adultos
}

\author{
F. OCHANDO CERDÁN, D. VEGA MENÉNDEZ, P. PACHECO MARTÍNEZ, \\ M. RAMOS FERNÁNDEZ, J. A. BLANCO CABELLOS ${ }^{1}$, P. HERNÁNDEZ \\ GRANADOS, C. LOINAZ SEGUROLA, A. QUINTANS RODRÍGUEZ \\ Servicios de Cirugía General y Aparato Digestivo y ${ }^{1}$ Radiodiagnóstico. \\ Fundación Hospital Alcorcón. Alcorcón. Madrid
}

URACHAL SINUS: AN UNUSUAL CAUSE OF RECURRENT UMBILICAL DISCHARGE IN ADULTS

\begin{abstract}
RESUMEN
El uracosinus es una anomalía congénita poco frecuente secundaria a la obliteración incompleta del uraco en su porción infraumbilical, que puede aparecer a cualquier edad.

Presentamos un paciente de 47 años que acudió al servicio de urgencias por supuración umbilical persistente que no había respondido al tratamiento médico (antibioterapia y curas). El TAC confirmó la existencia de un sinus del uraco con cambios de onfalitis. La cirugía consistió en la resección en bloque del mismo con onfalectomía. El postoperatorio transcurrió sin incidencias.
\end{abstract}

PALABRAS CLAVE: Uracosinus. Onfalitis recurrente. Malformaciones del uraco.

\begin{abstract}
Urachal sinus is a rare congenital anomaly due to incomplete closure the urachus in the umbilical region, it is very rare in adults. 47year-old male who arrived at our Emergency Department with recurrent umbilical discharge. Not response medical treatment (oral antibiotic and drainage). Abdominal computerized tomography scan confirmed the urachal sinus with omphalitis. Surgical complete excision with omphalectomy was performed. Any complications in the postoperative was observed.
\end{abstract}

KEY WORDS: Urachal sinus. Umbilical discharge. Urachal anomaly.

Ochando Cerdán F, Vega Menéndez D, Pacheco Martínez P, Ramos Fernández M, Blanco Cabellos JA, Hernández, Granados P, Loinaz, Segurola C, Quintans Rodríguez A. Uracosinus: una causa poco frecuente de onfalitis recurrente en adultos. An Med Interna (Madrid) 2006; 23: 329-330.

\section{INTRODUCCIÓN}

La onfalitis del adulto suelen ser localizada, de evolución tórpida y crónica, que generalmente, se correlaciona con la falta de una buena higiene del ombligo. A diferencia de la onfalitis del recién nacido que se produce por contaminación del cordón umbilical durante su sección o por deficientes cuidados postoperatorios y que conlleva una alta tasa de morbimortalidad debida a la diseminación de los gérmenes causantes (Staphilococcus aureus y Streptococcus beta hemolítico) por los vasos linfáticos y sanguíneos de la zona.

El tratamiento consiste en una adecuada higiene del ombligo, junto con antibioterapia si existe repercusión sistémica y drenaje quirúrgico con desbridamiento si existen colecciones purulentas asociadas.

La presencia de una onfalitis recurrente en un adulto con una buena higiene del ombligo, debe obligarnos a descartar la existencia de una patología subyacente, generalmente un cierre incompleto del uraco, aunque pueden existir otras causas (tumor primario o metastásico, quiste epidérmico umbilical, hernia umbilical, endometriosis, etc.) $(1,2)$.

\section{CASO APORTADO}

Paciente de 47 años que acudió al servicio de urgencias por supuración persistente umbilical y dolor, sin asociar fiebre u otros síntomas. El paciente había estado con antibioterapia (cloxacilina) y curas en su centro de salud sin ninguna mejoría. Los cultivos de la secreción umbilical fueron negativos. En la exploración se objetivaba supuración umbilical y empastamiento a nivel infraumbilical-linea media de aproximadamente $4 \mathrm{~cm}$, con celulitis asociada. El abdomen era blando y depresible, sin peritonismo. La analítica fue normal (no leucocitosis). Se realizó una ecografía que evidenció una colección infraumbilical, localizada en el plano muscular entre los dos rectos abdominales, de aproximadamente 27 x 16 x $30 \mathrm{~mm}$, de ecogenicidad heterogénea, que se comunicaba superficialmente con el ombligo. Se completó el estudio con un TAC, donde se apreciaron cambios inflamatorios infraumbilicales y una colección de unos $3 \mathrm{~cm}$,

Trabajo aceptado: 17 de enero de 2006 
con componente granulomatoso, que correspondía a un sinus del uraco con cambios asociados de onfalitis. Se objetivaba distal a ella, el ligamento del uraco que conecta con vejiga, obliterado (Fig. 1). La cirugía consistió en la resección en bloque del uracosinus con onfalectomía (Fig. 2). El postoperatorio transcurrió sin incidencias. La anatomía patológica descartó malignidad. No datos de recidiva tras 18 meses de seguimiento.

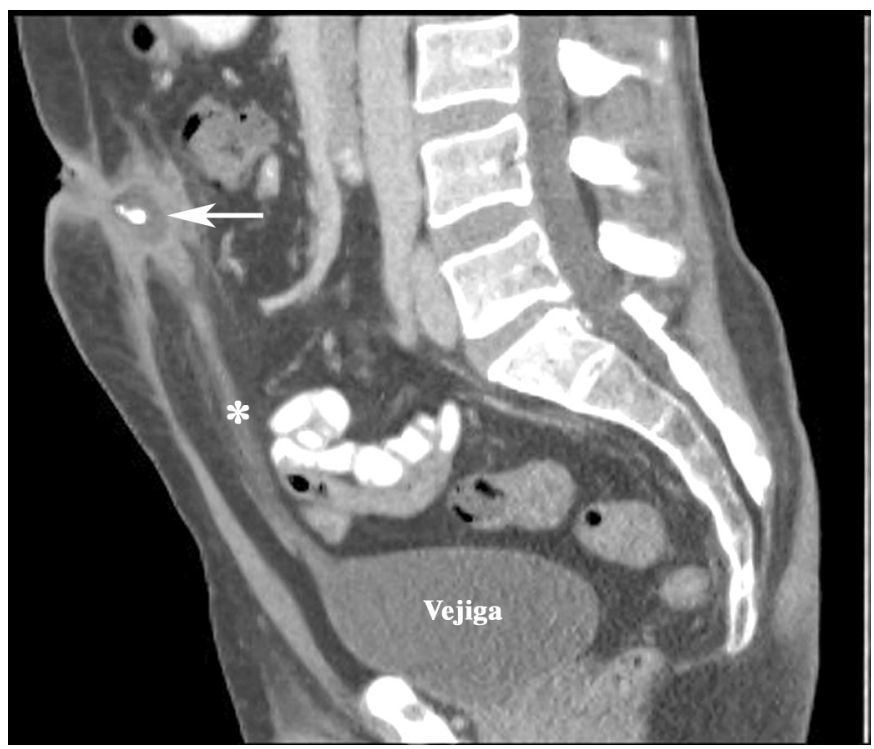

Fig. 1. TAC (reconstrucción sagital): Uracosinus (flecha). Uraco (asterisco).

\section{DISCUSIÓN}

El uraco es un conducto que comunica la vejiga con el ombligo en el feto y normalmente se oblitera en el momento del nacimiento (3).

Se han descrito diferentes tipos de anomalías congénitas del uraco (4), en función del grado y lugar de obliteración: uraco permeable $(50 \%)$ (determina la existencia de una fístula vesicoumbilical, que comunica vejiga con ombligo y ocasiona la salida de orina por el ombligo al estar permeable el uraco en todo su trayecto), quiste del uraco (30\%) (fallo de obliteración

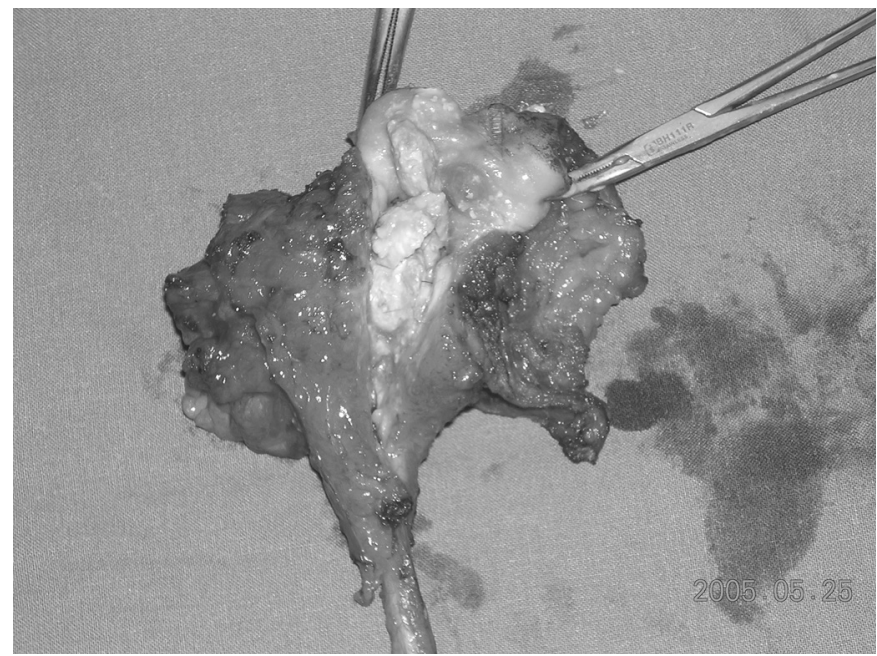

Fig. 2. Pieza quirúrgica.

en su porción media por lo que no comunica ni con vejiga ni con ombligo), uracosinus (15\%) (fallo de obliteración en su porción proximal, comunica con ombligo pero no con vejiga) y divertículo vesicouracal $(5 \%)$ (fallo de obliteración en su porción distal, comunica con vejiga pero no con ombligo).

El uracosinus o sinus del uraco, por tanto, es una anomalía congénita secundaria a la obliteración incompleta del uraco en su porción infraumbilical, que puede aparecer a cualquier edad. Clínicamente se manifiesta por supuración umbilical recurrente, junto con eritema, dolor leve o masa periumbilical (5). La ultrasonografía y el TAC ayudan en el diagnóstico, al permitir identificar las diferentes anomalías del uraco en la pared abdominal anterior (6). En ocasiones puede realizarse una fistulografía para confirmarlo (5). Se han descrito casos de malignización, lo cual apoyaría la exéresis quirúrgica del mismo (7). El tratamiento es quirúrgico y consiste en la resección en bloque del ombligo y el uracosinus. En los últimos años se ha incorporado el abordaje laparoscópico en su tratamiento (8).

En definitiva, ante una supuración crónica umbilical que no responda a las medidas higiénicas y antibioterapia, debemos pensar en la existencia de una causa subyacente, generalmente, un cierre incompleto del conducto uraco.

\section{Bibliografía}

1. McClenathan J. Umbilical epidermoid cyst: an unusual cause of umbilical symptoms. Can J Surg 2002; 45: 303

2. Rodriguez JM, Sanz Pelaez O, Santan L, Rey A. Suarez Ortega S, Betancor Leon P. The Sister Joseph's nodule-like manifestation of carcinoma of unknown origin: presentation of one case. An Med Interna (Madrid) 2005; 22: 285-287

3. Cappele O, Sibert L, Descargues J, Delmas V, Grise P. A study of the anatomic features of the duct of the urachus. Surg Radiol Anat 2001; 23: 229-235.

4. Allen J, Song J, Velcek F. Acute presentation of infected urachal cysts: case report and review of diagnosis and therapeutic interventions. Pediatr

Emerg Care 2004; 20: 108-111.

5. Oulasaiad M, Kamili E, Hakkoum J, Maksi B, Amal S, Akhdari N. Urachal sinus. Report of 3 cases. Prog Urol 2005; 15: 501-504.

6. Yu JS, Kim KW, Lee HJ, Lee YJ, Yoon CS, Kim MJ. Urachal remnant diseases: spectrum of CT and US findings. Radiographics 2001; 21: 451461.

7. Azurmendi Sastre V, Llarena Ibarguren R, Lozano Ortega JL, Martín Bazaco J, Pertusa Pena C. Urachal cyst. Current status. Arch Esp Urol 2003; 56: 999-1004.

8. Yohannes P, Bruno T, Pathan M, Baltaro R. Laparoscopic radical excision of urachal sinus. J Endourol 2003; 17: 475-479. 